\title{
Correlated electronic structure of Fe in bulk Cs and on a Cs surface
}

\author{
M. Costa, ${ }^{1,2}$ P. Thunström, ${ }^{3}$ I. Di Marco, ${ }^{2}$ A. Bergman, ${ }^{2}$ A. B. Klautau, ${ }^{4}$ A. I. Lichtenstein, ${ }^{5}$ M. I. Katsnelson, ${ }^{6}$ and O. Eriksson ${ }^{2}$ \\ ${ }^{1}$ Instituto de Física, Universidade Federal Fluminense, 24210-346 Niterói, Rio de Janeiro, Brazil \\ ${ }^{2}$ Department of Physics and Astronomy, Division of Materials Theory, Uppsala University, Box 516, SE-75120 Uppsala, Sweden \\ ${ }^{3}$ Institute for Solid State Physics, TU Wien, Wiedner Hauptstraße 8-10/E138, 1040 Wien, Austria \\ ${ }^{4}$ Departamento de Física, Universidade Federal do Pará, Belém, PA, Brazil \\ ${ }^{5}$ Institut für Theoretische Physik, Universität Hamburg, Jungiusstrasse 9, D-20355 Hamburg, Germany \\ ${ }^{6}$ Radboud University Nijmegen, Institute for Molecules and Materials, Heyendaalseweg 135, 6525AJ Nijmegen, The Netherlands
}

(Received 29 January 2013; published 27 March 2013)

\begin{abstract}
We have investigated the spectral properties of Fe impurities in a Cs host, for both surface and bulk systems, by means of a combination of density functional theory in the local density approximation and dynamical mean-field theory (LDA + DMFT). The effective impurity model arising in LDA + DMFT was solved via two different techniques, i.e., the Hubbard I approximation and the exact diagonalization. It is shown that noticeable differences can be seen in the unoccupied part of the spectrum for different positions of Fe atoms in the host, despite the fact that hybridization between $\mathrm{Fe} d$-states and $\mathrm{Cs}$ is low. Our calculations show good agreement with the experimental photoemission spectra reported by Carbone et al. [Carbone, Veronese, Moras, Gardonio, Grazioli, Zhou, Rader, Varykhalov, Krull, Balashov, Mugarza, Gambardella, Lebègue, Eriksson, Katsnelson, and Lichtenstein, Phys. Rev. Lett. 104, 117601 (2010)].
\end{abstract}

DOI: 10.1103/PhysRevB.87.115142

PACS number(s): 31.15.A-, 71.27.+a, 75.30.Hx

\section{INTRODUCTION}

The magnetic properties of most elements can be drastically modified by combining them to form compounds. An example of this is the ferromagnetic compound $\mathrm{ZrZn}_{2}$, which is formed by two elements that in pure form do not display any ordered magnetism. ${ }^{1}$ Another example is $\mathrm{YCo}_{2}$, a nonmagnetic compound (with a metamagnetic phase transition) ${ }^{2}$ containing an element (Co) that in pure form is a ferromagnet with significant saturation moment and ordering temperature. Another way to influence the magnetic properties of a material is to form inclusions of one element in a host material. An example that stands out here is Fe impurities in a Pd host, where the normally nonmagnetic $\mathrm{Pd}$ host becomes strongly spin polarized due to the proximity to the magnetic $\mathrm{Fe}$ atom. ${ }^{3}$ In this case a large number of $\mathrm{Pd}$ atoms become spin polarized, causing every $\mathrm{Fe}$ atom to be associated with a large moment of 9-12 $\mu_{B}$.

Recently much attention has been devoted to Fe impurities in Cs and other alkali metals. The reason for this interest is that Cs, according to the analysis of Wigner ${ }^{4}$ and Overhauser, ${ }^{5}$ is close to a magnetic state, since it has a very small electron concentration (the $r_{s}$ value is 6.6). However, more recent quantum Monte Carlo simulations ${ }^{6}$ show that these older theories greatly overestimate the tendency to form a ferromagnetic instability in homogeneous electron gas and that the bcc phase of $\mathrm{Cs}$ is in fact far from this instability. Nevertheless one may speculate that inclusion of small amounts of magnetic elements in $\mathrm{Cs}$, e.g., Fe or Co, could provide an exchange field that would push the host material to a magnetic ground state. Moreover, similarly to what happens for $\mathrm{Pd}$, magnetic inclusions in $\mathrm{Cs}$ could generate a cloud of spin-polarized atoms around the magnetic impurities. This idea was indeed tested experimentally in a series of experiments of $\mathrm{Fe}$ and $\mathrm{Co}$ in $\mathrm{Cs},{ }^{7-10}$ and large moments were reported. It was, however, argued that these large moments were not due to a polarization of the Cs host but more due to the $3 d$ shell assuming an atomiclike electronic configuration for the $\mathrm{Fe}$ and $\mathrm{Co}$ impurities, with atomic moments of $6 \mu_{B}$ /atom. $^{11,12}$ In fact, first-principles calculations, based on the density functional theory in the local density approximation (DFT-LDA), support a very small polarization of the Cs host atoms. $^{12,13}$ Nevertheless, experiments for Fe- and Co-doped $\mathrm{Rb}$ and $\mathrm{K}$ (Ref. 10) point to measured moments that are too large to be explained by atomic theory.

These materials have also been subjected to spectroscopical studies in the form of Fe atoms adsorbed on the Cs surface. ${ }^{14}$ The investigations involved both an experimental part and a theoretical part, the latter being based on the combination of DFT-LDA and dynamical mean-field theory, a computational scheme usually addressed as LDA + DMFT, ${ }^{15}$ in order to have a proper description of the correlated electronic structure. From this work it was concluded that $\mathrm{Fe}$ in $\mathrm{Cs}$ is a rather weakly hybridizing system, in which the $3 d$ states form essentially a localized/atomic electronic configuration, a conclusion which is in line with the suggestions of Refs. 11 and 12. In the present paper we provide a comprehensive LDA + DMFT study of the correlated electronic structure of $\mathrm{Fe}$ in $\mathrm{Cs}$, focusing on different positions of the Fe impurities in the host: substitutional impurity in the bulk $\mathrm{Cs}$, adsorbed on top of a $\mathrm{Cs}(001)$ surface and in an interstitial subsurface site.

\section{METHODS AND COMPUTATIONAL DETAILS}

We have performed simulations by means of a full potential linear muffin tin (FP-LMTO) code ${ }^{16,17}$ whose LDA + DMFT implementation has been thoroughly described elsewhere. ${ }^{18,19}$ In the LDA + DMFT scheme the lattice problem is mapped onto the problem of an impurity embedded in a fermionic bath, which reduces the complexity of the problem by freezing the spatial fluctuations but including all the dynamical quantum fluctuations. As a result of the mapping into an effective single 
impurity model, the DMFT solution is local in character, which becomes exact in the limit of infinite coordination number.

The effective impurity problem can be solved through different techniques. The choice of the solver is dependent on the system under consideration, on the properties one wants to calculate, and on the desired accuracy. In this work the Hubbard I approximation (HIA) ${ }^{20}$ and the exact diagonalization $(\mathrm{ED})^{21}$ solvers, as implemented in Ref. 22, were used. The HIA is suited for very localized systems, where the hybridization of the atomic impurity with the fermionic bath can be totally neglected. This assumption is generally true for $f$ states, and sometimes also for $d$ states, usually in compounds like complex oxides. The ED solver can be considered as the natural extension of the HIA: the hybridization with the fermionic bath is not neglected but approximated by means of a few bath orbitals. The Hamiltonian that describes the local problem becomes

$$
\begin{aligned}
\hat{H}= & \sum_{i j}\left(\hat{H}_{i j}^{\mathrm{LDA}}-\hat{H}_{i j}^{\mathrm{DC}}\right) \hat{c}_{i}^{\dagger} \hat{c}_{j}+\sum_{m} \epsilon_{m} \hat{c}_{m}^{\dagger} \hat{c}_{m} \\
& +\frac{1}{2} \sum_{i j k l} U_{i j k l} \hat{c}_{i}^{\dagger} \hat{c}_{j}^{\dagger} \hat{c}_{k} \hat{c}_{l}+\sum_{i m}\left(V_{i m} \hat{c}_{i}^{\dagger} \hat{c}_{m}+\text { H.c. }\right)
\end{aligned}
$$

The indices $i, j, k$, and $l$ label the impurity correlated orbitals, e.g., the Fe $d$ states, while $m$ labels the bath orbitals, e.g., the states derived from the Cs $s p$ states. The $H_{i j}^{\mathrm{LDA}}$ is obtained from the full LDA Hamiltonian projected on the correlated orbitals. The third term of Eq. (1) describes the local electron-electron interaction, and the $U_{i j k l}$ matrix can be written in terms of the Slater integrals $\left(F^{n}, n=0,2,4\right.$ for $d$ electrons) times the Gaunt coefficients (for instance as described in Ref. 23). The parameters $\epsilon_{m}$ represent the energy positions of the bath orbitals, while $V_{i m}$ are the hybridization strengths. In the HIA solver the hybridization is totally neglected, which corresponds to setting $\epsilon_{m}$ and $V_{i m}$ in Eq. (1) to zero. In this case bath and localized states are decoupled, and the problem is atomiclike. ${ }^{20}$ In the ED the parameters defining the bath orbitals are chosen by means of a numerical fitting, described in detail in Ref. 22. Once the Hamiltonian in Eq. (1) has been set up, the problem can be solved exactly by numerical diagonalization. From the eigenvalues and eigenvectors of $H$ one can finally obtain the local Green's function (and related properties) through the Lehman representation. Before describing the computational details, the double counting term $H_{i j}^{\mathrm{DC}}$ must be specified. This term is used to remove the contributions of the local Coulomb interaction $U_{i j k l}$ already contained in the LDA Hamiltonian. ${ }^{24}$ For the ED and HIA solver we find it convenient to use the photoemission data to set the double counting term, $H_{i j}^{\mathrm{DC}}=\mu^{\mathrm{DC}} \delta_{i j}$, where $\mu^{\mathrm{DC}}$ acts as a chemical potential, $\mu$ was then treated as a free parameter to get the main occupied peak at the correct position.

The method described above has been used for LDA + DMFT simulations of Fe impurities in Cs, using both the HIA and the ED solver. The Cs crystal structure is bcc with one atom in the basis. ${ }^{25}$ The $\mathrm{Cs}$ bulk and $\mathrm{Cs}(001)$ surface uses periodic boundary conditions and the smallest distance between the impurity and its image is $12 \AA$. The $\mathrm{Cs}(001)$ surface was modeled with seven slabs. Based on the size of $\mathrm{Fe}$ and $\mathrm{Cs}$ atoms the $\mathrm{Fe}$ adsorbed was set to $60 \%$ of the bulk value in the $z$ direction. A Monkhorst-Pack mesh of $7 \times 7 \times 7$ $(7 \times 7 \times 1)$ was used for the bulk (surface) calculations. The basis set used to expand the electronic density inside the muffin tins is constructed from a linear combination of radial functions multiplied by spherical harmonics $\left(l_{\max }=8\right)$. For the interstitial region a combination of Hankel and Neumann functions are used. The Slater parameters describing the $U_{i j k l}$ matrix were constructed from the Hubbard $U$ and Hund's $J$ parameters by following the prescription of Ref. 26. The Hubbard parameter $U\left(F^{0}\right)$ is very sensitive to the environment, and in a free atom can be as high as $30 \mathrm{eV}$. In a solid, due to the screening effect, $U$ ranges from 2 to $8 \mathrm{eV}$. As the aforementioned studies point to that $\mathrm{Fe}$ is in an essentially atomiclike environment, $U=8.0 \mathrm{eV}$ was used. The intratomic exchange parameter $J$ was set to 0.75 (for the $d^{6}$ configuration) and 0.82 (for the $d^{7}$ configuration) $\mathrm{eV}$.

\section{RESULTS}

As mentioned in the Introduction, it has been a long debate concerning the nature of the electron ground state of Fe impurities in alkali hosts. The issue concerns whether Fe is in a $d^{6}$ or $d^{7}$ electronic configuration. Despite a very small hybridization of $\mathrm{Fe}$ with $\mathrm{Cs}$, a charge transfer from the electropositive host to the Fe impurity can occur. It is not possible to discriminate these $d^{6}$ and $d^{7}$ configurations by the value of the effective moment, since they are very close. Gambardella et al. ${ }^{27}$ claimed that $\mathrm{Fe}$ impurities in $\mathrm{K}$ are in a $d^{7}$ configuration. They used $\mathrm{x}$-ray absorption spectroscopy (XAS) measured data and compared them to atomic multiplet calculations done by van der Laan and Thole. ${ }^{28}$ Because there are some discrepancies in their analysis, our first aim is to determine the ground state electronic configuration of the $\mathrm{Fe}$ impurity in a $\mathrm{Cs}$ host. The photoemission spectra of $\mathrm{Fe}$ impurities in a Cs host do not differ very much from the $\mathrm{K}$ data, as was shown by Carbone et al. ${ }^{14}$ Hence we believe

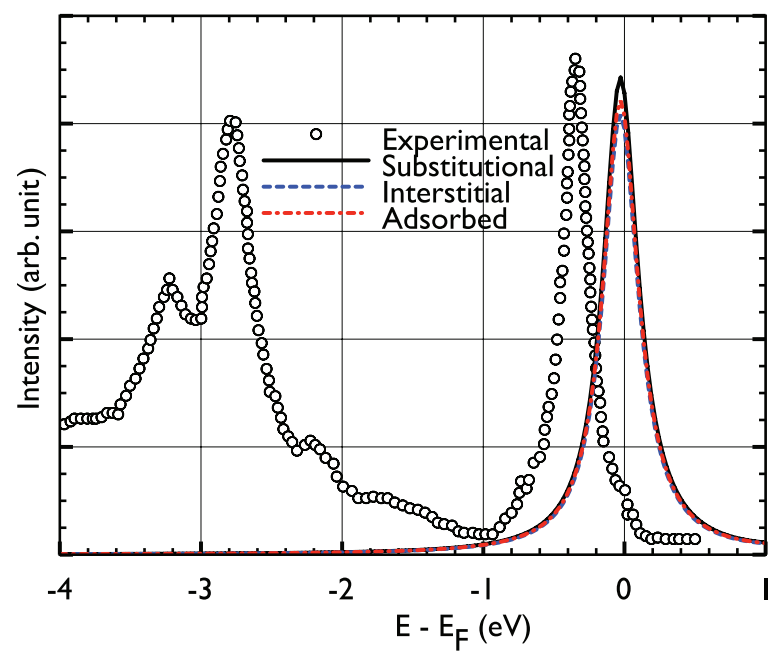

FIG. 1. (Color online) LDA projected density of states for the Fe $d$ states at different positions: substitutional in the Cs bulk (solid black line), interstitial in the first sublayer of the $\mathrm{Cs}(001)$ surface (dashed red line), and adsorbed on top of the $\mathrm{Cs}(001)$ surface (dotted-dashed blue line). The experimental photoemission spectrum (empty circles) from Ref. 14 is also shown. 

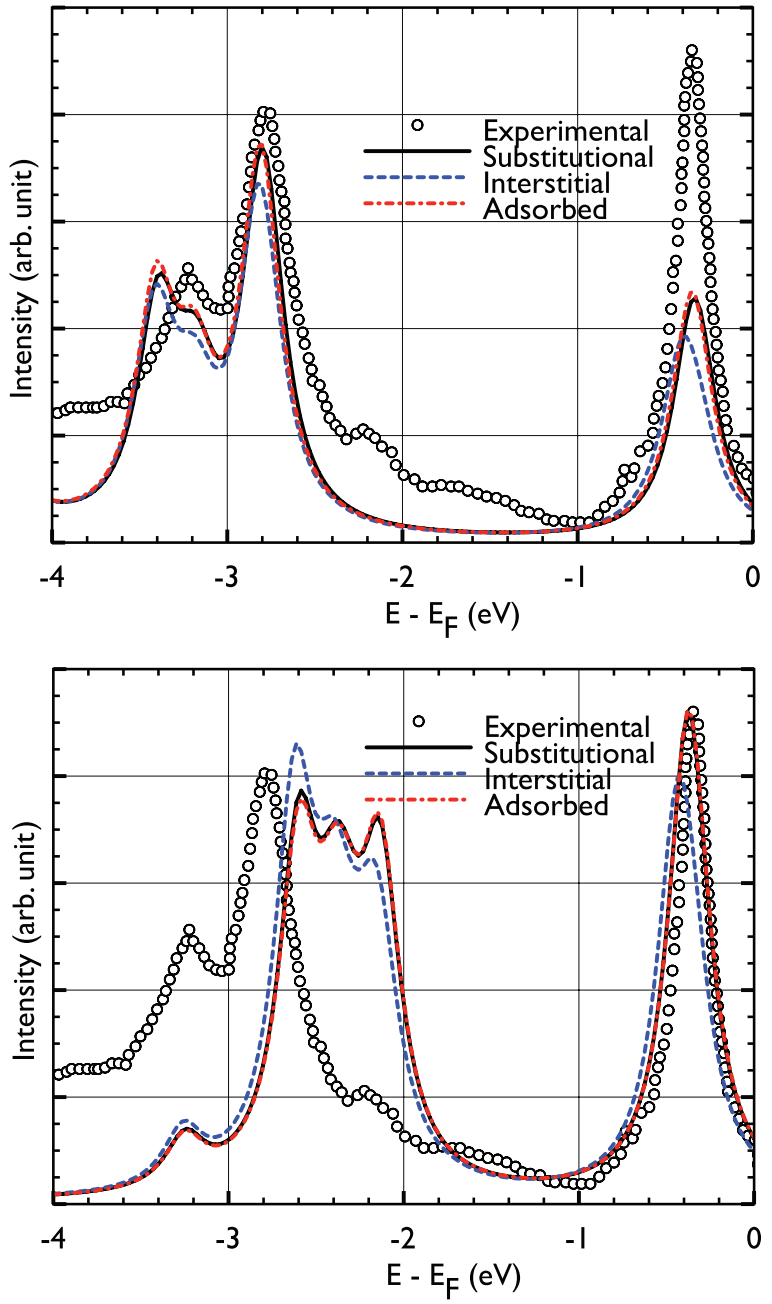

FIG. 2. (Color online) HIA PDOS for the Fe $d$ states at different positions: substitutional in the Cs bulk (solid black line), interstitial in the first sublayer of the $\mathrm{Cs}(001)$ surface (dashed red line), and adsorbed on top of the $\mathrm{Cs}(001)$ surface (dotted-dashed blue line). The top (bottom) panel is the $d^{6}\left(d^{7}\right)$ configuration. The experimental photoemission spectrum (empty circles) from Ref. 14 is also shown.

that our analysis for $\mathrm{Fe}$ in $\mathrm{Cs}$, is also valid for the case of $\mathrm{Fe}$ in $\mathrm{K}$.

First we compared the LDA calculation of the $d$ states projected density of states (PDOS) for Fe impurities in a Cs host to experimental data from photoemission spectroscopy reported by Carbone et al. ${ }^{14}$ Figure 1 shows the PDOS for Fe $d$ states in the LDA for the substitutional, interstitial, and adsorbed impurity. It is clear that the LDA does not capture the measurement. The experimental spectrum exhibits multipletlike features, while the LDA PDOS has a single peak centered on the Fermi level. The predicted spectral properties do not show any correspondence with the experimental data.

In the top panel of Fig. 2, which corresponds to a calculation of the $d^{6}$ configuration, the same experimental spectrum is compared to the PDOS for the Fe $d$ states calculated with the $\mathrm{LDA}+$ DMFT scheme with the HIA solver. It is possible to recognize that these results are in good agreement with the

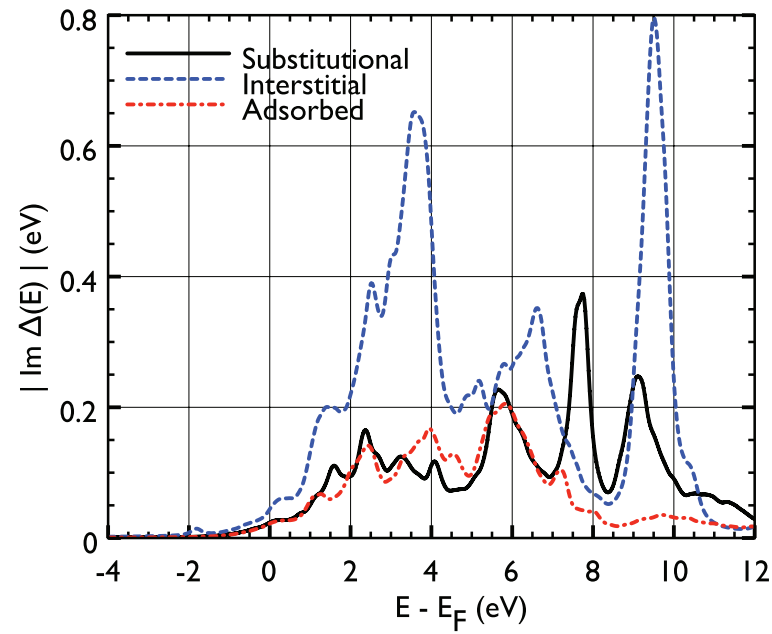

FIG. 3. (Color online) Strength of the hybridization for the Fe $d$ states in a LDA calculation for different positions: substitutional in the Cs bulk (solid black line), interstitial in the first sublayer of the $\mathrm{Cs}(001)$ surface (dotted-dashed red line) and adsorbed on top of the Cs(001) surface (dashed blue line).

experimental photoemission spectrum and all major multiplet features are captured. The first peak (at $-0.35 \mathrm{eV}$ ) is used to set the double counting term, as explained above, and by construction should be on top of the experimental one. The second experimental peak, which has a weak intensity, is at around $-2.3 \mathrm{eV}$ and is the only feature absent from our calculation. The third peak, at $-2.8 \mathrm{eV}$, shows a very good correspondence between experiment and theory. Finally the position of the fourth peak is well reproduced by the theory, at around $-3.2 \mathrm{eV}$. Overall, the energy separation between the calculated peaks is in good agreement with experiment. Probably a better agreement can be obtained by tweaking the exact values of the Slater parameters $F^{2}$ and $F^{4}$, but this procedure would not lead to any additional significant information and therefore was not done. As for the calculations of the $d^{7}$ configuration, lower panel of Fig. 2, despite capturing the multiplet features, the overall agreement is not as good as for the $d^{6}$ configuration; nevertheless the second peak that is missed in the $d^{6}$ PDOS can be seen here despite its intensity being severely overestimated.

We now proceed with an analysis of the effects due to the hybridization of the atomic impurity with the surrounding orbitals. In Fig. 3 we show the strength of the hybridization of the Fe $d$ states, in the $d^{6}$ configuration, obtained from the trace of the imaginary part of the hybridization function ${ }^{22}$ over all orbitals. As expected, the hybridization is very small for all the possible positions of the impurity, especially in the occupied part of the spectrum. In the unoccupied part, the $\mathrm{Fe}$ at the interstitial position shows the strongest hybridization, while the $\mathrm{Fe}$ at the adsorbed position shows the weakest. The effects of the hybridization on the spectrum are clear when LDA + DMFT simulations are performed with the ED solver. The bath states reproducing the Fe hybridization, which are obtained through the aforementioned fitting algorithm, ${ }^{22}$ are fully specified by the parameters $\epsilon_{m}$ and $\mathrm{V}_{m}$. These parameters are reported in Table I. 
TABLE I. Hybridization energies $\epsilon_{m}(\mathrm{eV})$ and strength $\mathrm{V}_{m}$ used in the exact diagonalization solver. Ten bath orbitals were included.

\begin{tabular}{lcc}
\hline \hline & $\epsilon_{m}(\mathrm{eV})$ & $\mathrm{V}_{m}\left(10^{-2} \mathrm{eV}\right)$ \\
\hline Substitutional & 1.861 & 2.205 \\
& 4.262 & 1.986 \\
& 6.301 & 2.034 \\
Interstitial & 2.675 & 4.018 \\
& 2.733 & 3.352 \\
& 3.114 & 4.192 \\
Adsorbed & 4.256 & 5.099 \\
& 2.818 & 1.421 \\
& 3.281 & 2.148 \\
& 4.241 & 2.438 \\
& 5.829 & 2.321 \\
\hline \hline
\end{tabular}

The occupied part of the ED spectrum for three possible Fe positions is reported in Fig. 4; for the $d^{6}$ configuration, the spectrum is very similar to the HIA spectrum. The only visible change is on the first peak the interstitial impurity becomes smaller, which is expected as the interstitial impurity presents the highest hybridization. Finally we have also investigated the unoccupied part of the spectrum for the $d^{6}$ configuration, as reported in Fig. 5. This was motivated by the fact that in this region the hybridizations, shown in Fig. 3, exhibit the largest contributions. For the HIA spectrum no significant difference can be seen for the three sites. This is expected since HIA neglects all hybridization of the $\mathrm{Fe} d$ states with the neighboring Cs atoms. For the ED spectrum a major difference between the adsorbed spectrum and the substitutional (interstitial) spectrum is observed. This can be understood in terms of the hybridization of the Fe impurity with the Cs. The hybridization couples the impurity orbitals (Fe) to the bath empty orbitals (Cs). This coupling opens

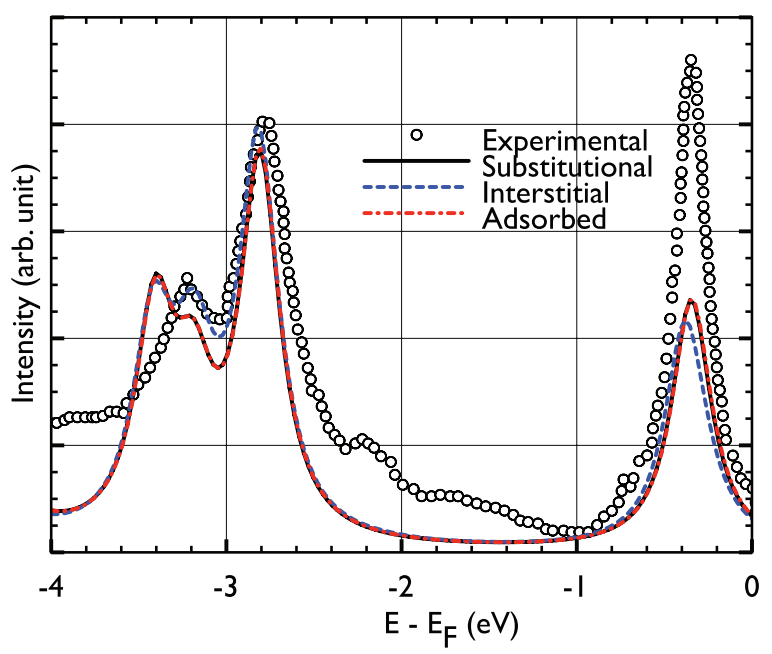

FIG. 4. (Color online) ED PDOS for the Fe $d$ states at different positions: substitutional in the Cs bulk (solid black line), interstitial in the first sublayer of the $\mathrm{Cs}(001)$ surface (dashed red line), and adsorbed on top of the $\mathrm{Cs}(001)$ surface (dotted-dashed blue line). The experimental photoemission spectrum (empty circles) from Ref. 14 is also shown.
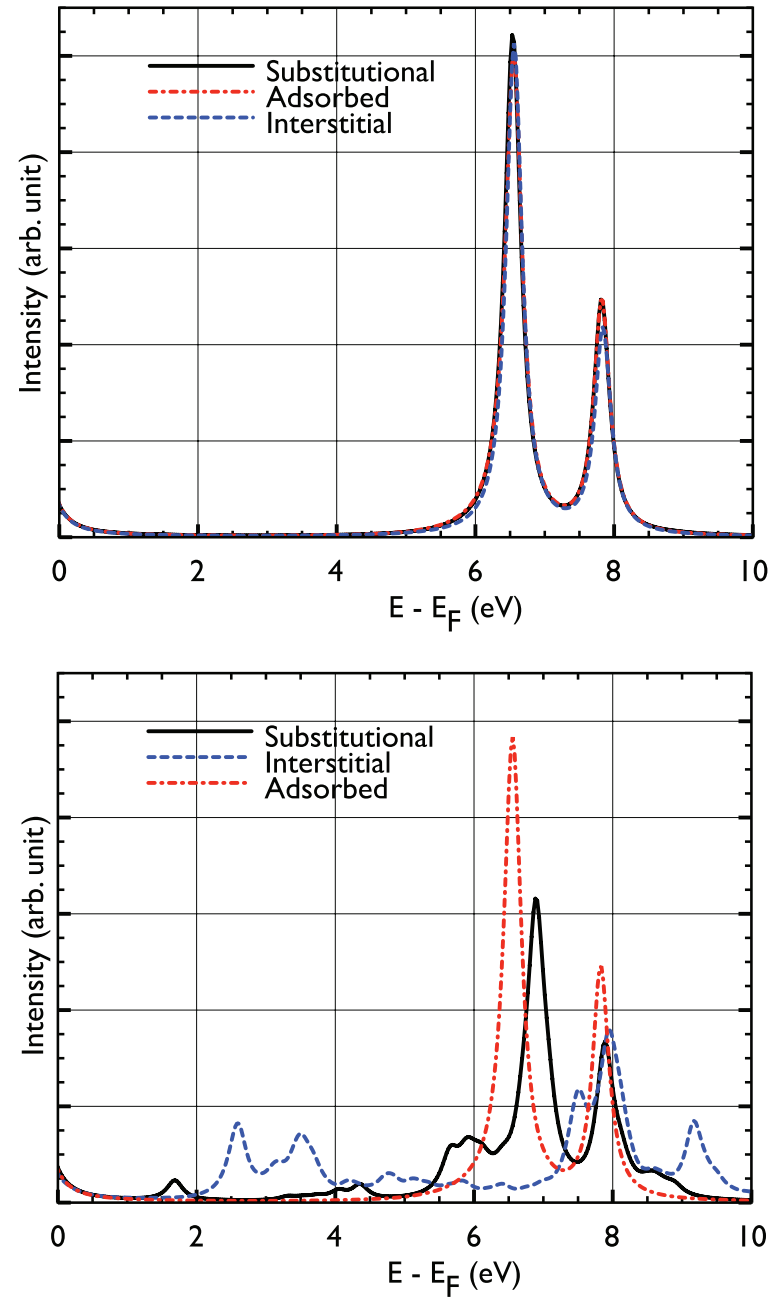

FIG. 5. (Color online) Top (bottom) panel shows the HIA (ED) unoccupied PDOS for the Fe $d$ states at different positions: substitutional in the Cs bulk (solid black line), interstitial in the first sublayer of the $\mathrm{Cs}(001)$ surface (dotted-dashed red line), and adsorbed on top of the $\mathrm{Cs}(001)$ surface (dashed blue line).

channels for electrons to hop from the impurity orbitals to the bath orbitals. The effect of this hopping is seen in the appearance of more peaks for the substitutional and interstitial sites in the unoccupied part of the spectrum.

\section{CONCLUSION}

We performed LDA + DMFT calculations to investigate the spectral properties of Fe impurities in $\mathrm{Cs}$, for both bulk and surface host. It was shown that pure LDA calculations lead to a very poor description of the spectral properties with respect to experimental photoemission data. In the LDA + DMFT scheme the HIA is very successful in reproducing the occupied part of the excitation spectrum. Our finding supports a $d^{6}$ electronic configuration for the $\mathrm{Fe}$ impurity in a Cs host, in contrast to previous suggestions of a $d^{7}$ configuration. ${ }^{14}$ From our calculations of the occupied spectrum it is not possible to see significant differences among the three positions that the impurity is most likely to occupy. These trends are confirmed also in the LDA + DMFT simulations with the ED solver, 
since the resulting occupied part of the spectrum is very similar for all the impurity sites investigated. However, we find that significant differences between the theoretical spectra for the different positions of the Fe impurity can be found in the unoccupied part. This suggests that one can use inverse photoemission spectroscopy, which probes the unoccupied electronic excitations, to identify in which site the impurity is located.

\section{ACKNOWLEDGMENTS}

Support by the Swedish Research Council (VR) is acknowledged, as is the European Research Council (Project No. 247062-ASD), the KAW Foundation, eSSENCE, STEM, and the Swedish National Infrastructure for Computing (SNIC). M.C. also acknowledges partial financial support by $\mathrm{CNPq}$ and CAPES (Brazil).
${ }^{1}$ S. Foner, E. J. McNiff, and V. Sadagopa, Phys. Rev. Lett. 19, 1233 (1967); S. Ogawa and N. Sakamoto, J. Phys. Soc. Jpn. 22, 1214 (1967).

${ }^{2}$ K. Schwarz and P. Mohn, J. Phys. F 14, L129 (1984).

${ }^{3}$ G. G. Low and T. M. Holden, Proc. Phys. Soc. 89, 119 (1966); G. Nieuwenhuys, Adv. Phys. 24, 515 (1975); M. Pärnäste et al., J. Phys.: Condens. Matter 19, 246213 (2007).

${ }^{4}$ E. Wigner, Trans. Faraday Soc. 34, 678 (1938).

${ }^{5}$ A. W. Overhauser, Adv. Phys. 27, 343 (1978).

${ }^{6}$ D. M. Ceperley and B. J. Alder, Phys. Rev. Lett. 45, 566 (1980).

${ }^{7}$ G. Bergmann, D. Frank, and D. Garrett, Eur. Phys. J. B 5, 345 (1998).

${ }^{8}$ H. Beckmann, T. Fulmer, D. Garrett, M. Hossain, and G. Bergmann, Phys. Rev. B 59, 7724 (1999).

${ }^{9}$ D. Riegel, H. J. Barth, L. Buermann, H. Haas, and C. Stenzel, Phys. Rev. Lett. 57, 388 (1986).

${ }^{10}$ M. Hossain and G. Bergmann, Eur. Phys. J. B 26, 7 (2002).

${ }^{11}$ M. Gruyters and D. Riegel, Phys. Rev. Lett. 85, 1582 (2000).

${ }^{12}$ P. Mohn, P. Weinberger, B. Ujfalussy, O. Eriksson, G. Gutieres, R. Ahuja, and B. Johansson, Phys. Rev. Lett. 85, 1583 (2000).

${ }^{13}$ G. Y. Guo, Phys. Rev. B 62, R14609 (2000).

${ }^{14}$ C. Carbone, M. Veronese, P. Moras, S. Gardonio, C. Grazioli, P. H. Zhou, O. Rader, A. Varykhalov, C. Krull, T. Balashov, A. Mugarza, P. Gambardella, S. Lebègue, O. Eriksson, M. I. Katsnelson, and A. I. Lichtenstein, Phys. Rev. Lett. 104, 117601 (2010).

${ }^{15}$ For a review see G. Kotliar, S. Y. Savrasov, K. Haule, V. S. Oudovenko, O. Parcollet, and C. A. Marianetti, Rev. Mod. Phys. 78, 865 (2006); K. Held, Adv. Phys. 56, 829 (2007); M. I. Katsnelson,
V. Yu. Irkhin, L. Chioncel, A. I. Lichtenstein, and R. A. de Groot, Rev. Mod. Phys. 80, 315 (2008).

${ }^{16}$ J. M. Wills and B. R. Cooper, Phys. Rev. B 36, 3809 (1987).

${ }^{17}$ J. M. Wills, M. Alouani, P. Andersson, A. Delin, O. Eriksson, and A. Grechnev, Full-Potential Electronic Structure Method: Energy and Force Calculations with Density Functional and Dynamical Mean Field Theory, Springer Series in Solid-State Sciences, Vol. 167 (Springer, New York, 2010).

${ }^{18}$ A. Grechnev, I. Di Marco, M. I. Katsnelson, A. I. Lichtenstein, J. M. Wills, and O. Eriksson, Phys. Rev. B 76, 035107 (2007).

${ }^{19}$ O. Grånäs, I. Di Marco, P. Thunström, L. Nordström, O. Eriksson, T. Björkman, and J. M. Wills, Comput. Mater. Sci. 55, 295 (2012).

${ }^{20}$ A. I. Lichtenstein and M. I. Katsnelson, Phys. Rev. B 57, 6884 (1998).

${ }^{21}$ M. Caffarel and W. Krauth, Phys. Rev. Lett. 72, 1545 (1994).

${ }^{22}$ P. Thunström, I. Di Marco, and O. Eriksson, Phys. Rev. Lett. 109, 186401 (2012).

${ }^{23}$ R. M. Martin, Electronic Structure: Basic Theory and Practical Methods (Cambridge University Press, Cambridge, UK, 2004).

${ }^{24}$ M. Karolak, G. Ulm, T. Wehling, V. Mazurenko, A. Poteryaev, and A. Lichtenstein, J. Electron Spectrosc. Relat. Phenom. 181, 11 (2010).

${ }^{25}$ C. Ortiz, O. Eriksson, and M. Klintenberg, Comput. Mater. Sci. 44, 1042 (2009).

${ }^{26}$ I. V. Solovyev, P. H. Dederichs, and V. I. Anisimov, Phys. Rev. B 50, 16861 (1994).

${ }^{27}$ P. Gambardella, S. S. Dhesi, S. Gardonio, C. Grazioli, P. Ohresser, and C. Carbone, Phys. Rev. Lett. 88, 047202 (2002).

${ }^{28}$ G. van der Laan and B. T. Thole, Phys. Rev. B 43, 13401 (1991). 